\title{
The Role of Hope and Social Support on Resilience in Cancer patients
}

\author{
Juhi Vartak ${ }^{1}$ \\ ${ }^{1}$ Post Graduate Student, Department of Psychology, Maniben Nanavati Women's College, Mumbai \\ E-mail-jus6999@gmail.com
}

\begin{abstract}
Resilience as a construct in the recovery from cancer has been a widely studied area. The present study aimed to investigate the impact of hope and social support on cancer patient's resilience to deal with their illness. This was a quantitative research and data was collected using researcher administered questionnaire surveys. Participants comprised of 115 cancer patients. The baseline questionnaire was administered to assess the social support, hope and resilience in the patient. Several statistical tests including Two-way ANOVA un-weighted data analysis, frequency analysis, correlation and regression tests were performed. A Pearson's Product Moment correlation reported all three variables are positively correlated with each other. A simple linear Regression analysis was carried out with resilience as the independent variable and hope and support as independent variables. Results indicate that both hope and social support have a positive and statistically significant impact on the resilience of cancer patients ( $p$ $<0.05$ for hope and social support individually). These findings raised the possibility that intervention at an early stage can increase the resilience and improve the mental health of cancer patients.
\end{abstract}

Key words: social support, cancer, hope, resilience, cancer patients.

\section{INTRODUCTION}

Cancer is a leading cause of death around the world. In 2007, cancer claimed the lives of about 7.6 million people in the world [1]. This diagnosis of cancer is devastating to the society; it is not only affecting the patient but also their families. Close friends and relatives who are directly in contact with the cancer survivors are the secondary survivors who are affected by the diagnosis of their relatives [2].

Family members of cancer patients are often termed as 'secondary survivors' that are often profoundly affected by the cancer diagnosis of a loved one [3]. In another study it was revealed that though there is an additional responsibilities when the family member is suffering and getting treated for cancer, this responsibility was not perceived as burden to the family members. Interview data from the participants of this study identified hope and resilience impacting their ability to provide care. These data justified further examination of the concepts of hope and resilience among persons newly diagnosed with cancer and a family member and effect of social support [4].

Hope and social support increases the resilience but this change over time, from the perspective of a patient who have been diagnosed with cancer. 
Researchers have documented physical, emotional, and psychological effects of a cancer diagnosis for the patient with cancer. The importance of hope social support and resilience on cancer survivorship has received little research attention. A literature review identified two studies, which assessed hope and resilience, for the person with cancer and family caregiver, at the time of initial diagnosis. Moreover it also stated that the timeframe of initial cancer diagnosis is important, postulating that this is "when psychosocial dimensions may be more vulnerable to the stress of coping with the new diagnosis and life changes entailed by cancer treatment" [5]. Therefore, hope and resilience are critical concepts to understand. Another research identified hope and resilience as influencing the capability of the person with cancer and their family to cope with the effects of the cancer diagnosis and treatment. According to him, instruments that measure social support, hope and resilience are available and is specific for the cancer population [6].

\section{Resilience}

Resilience has become a focus of research during the last decades in the behavioral sciences [7]. While resilience has been defined as resistance to illness, adaptation and thriving, the ability to bounce back or recover from stress is closest to its original meaning. Previous resilience measures assess resources that may promote resilience rather than recovery, resistance, adaptation, or thriving "resile," which means, "to bounce or spring back" [8]. Resilience is the ability to successfully cope with change or misfortune. For centuries, cancer has been considered a deadly disease. However, with advancement in medical science, early intervention can help cure cancer in many cases. Nevertheless, most patients feel devastated and frustrated when their cancer is first diagnosed, and many still must fight a very difficult battle against the disease [9].

\section{Hope}

Hope is often considered an important factor in patients' personal adjustments during times of loss, uncertainty, and suffering [10]. However, the concept of hope has been challenging to research because of its ambiguous nature, its blend of intangibility and reality, and various individual interpretations of its meaning. Because of fear about an uncertain future, patients often begin looking to additional sources for support in handling the stress of their disease. Hope is considered aneffective coping strategy for patients with cancer, providing adaptive power to help them get through the difficult situation and achieve desired goals [11]. Data suggests that patients can develop a sense of helplessness or hopelessness when stress becomes overwhelming. This response is associated with higher rates of death, although the mechanism for this outcome is unclear. Emotional and social support can help patients learn to cope with psychological stress. Such support can reduce levels of depression, anxiety, and diseaseand treatment-related symptoms among patients [12].

\section{Social support}

Definitions of social support would range from, actual supportive acts which are exchanged by individuals to a Personality-like factor which is based in early interpersonal experiences that will influences into how an person views the likelihood of someone is supportive. Social support had been measured in the past in numerous ways. One of the frequent criticisms of social support researchers is lack of consensus about the definition. Social support is usually defined as the existence of people on whom we can rely, people who let us know that they care about, value, and love us. A broad definition of social support is the "resources provided by others" [13]. 
There is a distinction between existence of social relationships and the functions provided by these. The structure would be "how many friends, colleagues, family relationships" you have. The functional aspect would refer to what support do they provide. The social support of a person consists of the network of social relationship and the support from this social network. Social support provides emotional and psychological support that could enhance the hope and resilience in tackling adversity. A systematic review of resilience of seriously ill patients showed that Social support was highly predictive of, and associated with, resilience. Coping strategies such as positive cognitive appraisal, spirituality, active coping, and mastery were also associated with resilience. Resilience factors directly salient to physical illness such as self-care, adherence to treatment, health related quality of life, illness perception, pain perception, exercise adherence, and physical outcomes were also found [14].

Resilience is a subject of increasing research over the past decade due to its potential to impact on health outcome. A diagnosis of cancer is often regarded as a potential traumatic event with adverse consequences. Research frequently focuses on negative aspect of cancer diagnosis and treatment [15]. The psychological and social impact on the caregiver and the people associated with the patient is not known. A study showed that earlier work has indicated that family cohesion is strengthened by childhood cancer, with spouses being an important source of support for each other [16]. In another study it was revealed that the time duration of awareness of cancer plays a moderating role in the relation between the general dimension of quality of life and resiliency. In patients who were aware of their disease for over 12 months, the relationship between resiliency and the general dimension of quality of life was significant. In addition, high levels of resiliency showed a positive and significant relationship with physical dimension of life quality. While in patients who were aware of their illness for less than 12 months, resiliency scores showed a significant relation with emotional dimension of quality of life [17]. In a longitudinal study conducted, both the cancer patient and the family member were administered the test on hope at two time intervals; within 30 days of a cancer diagnosis and 30 days later. Study data included responses on the Herth Hope Index (HHI), the Resilience Scale (RS), and self-disclosed demographic information. It was hypothesized that individual HHI and RS scores would change over time. Results of this study indicated that hope and resilience for the person with cancer and the family member do change. These two concepts correlated for the person with cancer and the family member. This study identified the need for intervention(s) addressed at hope and resilience [18].

Researchers investigated the relationship between hope and coping among 120 adult patients undergoing chemotherapy in hospital, outpatient, and home settings. A significant relationship existed between the level of hope and the level of coping among participants in all three settings. Religious convictions and family support were significant factors in hope and coping regardless of setting. There is a profound effect on the family of the patient who suffers from cancer [19].

\section{METHODOLOGY}

The aim of this present study is to examine the effect of resilience of cancer patient and the effect of hope and social support in their life. There are two independent variables, hope and social support with two levels high and low. The dependent variable is the level of resilience. 


\section{Hypothesis}

There are four hypotheses to this study -

$\mathrm{Ho}_{1}$ : There is no significant Effect of Hope on Resilience.

$\mathrm{H} 1_{1}$ : There is a significant Effect of Hope on Resilience.

$\mathrm{HO}_{2}$ : There is no significant Effect of Social Support on Resilience.

$\mathrm{H}_{1}$ : There is a significant Effect of Social Support on Resilience.

$\mathrm{Ho}_{3}$ : There will be no significant interaction effect between hope and social support on resilience. Higher the hope and social support higher will be resilience.

$\mathrm{H}_{1}$ : There will be a significant interaction effect between hope and social support on resilience. Higher the hope and social support higher will be resilience.

In this study 'Hope' is considered to be an effective coping strategy for patients with cancer, providing adaptive power to help them get through the difficult situation and achieve desired goals. Hope is also considered as an important factor in patients' personal adjustments during times of treatment, uncertainty, and suffering. High Hope is positive thinking of recovery with treatment and acceptance of the disease as influencing the capability of the person to cope with the effects of the cancer diagnosis and treatment.

The other independent variable 'Social support' is defined as "someone who is always there for you." It refers to the extent to which individuals receives help and support from their families, friends, and relatives. Social support of a person consists of the network of social relationship and the support from this social support. The person's own experiences with this relationship and the support derived from them towards positive recovery. Social support prevents the individual from the harmful effects of the disease. Physical as well as psychological that enhances or reduces the hope to face new challenges of health and social situation.

Resilience as a dependent variable is defined as the ability to successfully cope with change or misfortune. Resilient individuals regain their balance and keep going despite adversity and misfortune. Resilience is defined as resistance to illness, adaptation and thriving, the ability to bounce back or recover from stress caused due to illness rather than fear change and challenge. Resilient persons are self-confident and understand their own strengths and abilities. The effect of hope and social support can make a remarkable difference in the resilience of the individual suffering from cancer.

Participants for this study include patients who were detected with cancer, patients who were undergoing therapy, and patients who have come for treatment or post treatment consultation. Participants were required to have Basic English speaking and writing skills as there were some terms in the questionnaire which will be difficult for non English speaking individuals to understand. All the participants were residents of Mumbai but this was not a criterion for selection. Only patientswho have been taking medicinal treatment since last 6 months were considered for this study.

In all 115 cancer patient undergoing treatment of cancer including chemotherapy, radiation or surgery were randomly selected and used as participants in the study. Informed and written consent was obtained from patients as well as the management of hospitals where the survey was carried out. The study excluded patients who were at the terminal stage of cancer or patients under Palliative care taking special medication. 


\section{Scales Used}

Demographic Questionnaire: Subjects were asked to complete a standard questionnaire about their Age, Education, Language Known or Spoken, work Information Marital Status, type, Stage and treatment for cancer.

Herth Hope Scale: The Herth Hope scale was used to determine the level of hope in the patient which consisted of six questions. The subjects were asked to rate their feelings over the past few weeks on a five-point likert scale. Results will indicate whether the patient has scored high or low level hope [20].

The Brief resilience scale: The Brief resilience scale was measured with the Brief resilience scale consisting of five point likert scale. The scale was created to Asses the ability to bounce back or recover from stress. The BRS is a reliable means of assessing resilience [21].

Perceived social support scale: The Multidimensional Scale of Perceived social support received by the patient.. A Perceived social support scale was administered to understand the degree of social support the patient has to help him/her overcome his fight with cancer. Addressing a different source of support, were identified and found to have strong factorial validity: (a) Family, (b) Friends, and (c) Significant Other [22].

\section{Statistical Analysis}

Two way ANOVA using the computerised software for Independent Sample and weighted-means analysis to examine the effects of hope and social support on the resilience of the cancer patient was done. Results indicating, difference with a probability of less than or equal to 0.05 were accepted as a significant.

\section{RESULTS}

For Hope: From the results we observe that the $p$-value for hope is 0.0407 which is less than 0.05 . So we Reject $\mathrm{Ho}_{1}$ and conclude that there is a significant effect of hope on resilience at $5 \%$ level of significance.

We also conclude that patients who have higher level of hope will have higher level of Resilience and atients who have lower level of hope will have lower level of resilience.

For Social Support: From the results we observe that the p-value for hope is 0.0064 which is less than 0.05 . So we Reject $\mathrm{Ho}_{2}$ and conclude that there is a significant effect of Social Support on resilience at $5 \%$ level of significance.

We also conclude that patients who have higher level of Social Support will have higher level of Resilience and patients who have lower level of Social Support will have lower level of resilience.

For Hope and Social Support: From the results we observe that the p-value for hope and social support is 0.7918 which is greater than 0.05 . So we Accept $\mathrm{Ho}_{3}$ and conclude that there is no significant interaction effect of Hope and Social Support on resilience at $5 \%$ level of significance. The Tukey HSD TEST result shows that there is a significant difference between the mean between the two rows and two columns since the $p$-value is less than the mean difference of the two rows and two columns i.e for rows mean difference $0.4598>0.33$ and column mean difference $0.596>0.34$ at $5 \%$ I.o.s.

The Correlational analysis for the responses was carried out. The output of the correlation test is given in the table below: 


\begin{tabular}{|l|l|l|l|}
\hline & Resilience & Hope & Support \\
\hline Resilience & 1 & & \\
\hline Hope & 0.397345 & 1 & \\
\hline Support & 0.468924 & 0.243112 & 1 \\
\hline
\end{tabular}

The Results indicate that all the three variables are positively correlated with each other. However, correlation coefficients are all less than 0.5 indicating that although correlation is positive but there is not high degree of correlation in the variables.

Regression analysis is a statistical tool for the investigation of relationships between variables. The regression model for this research is:

Resilience $=a_{0}+a_{1} *$ Hope $+a_{2} *$ Support

Where $a_{0}, a_{1}, a_{2}$ are coefficients of regression.

Adjusted R Square value of 0.2928 indicates that hope and support can explain up to $29.28 \%$ variance in the resilience of cancer patients. In this model p-value for both hope and support are less than 0.05 indicating a statistically significant relationship between independent variables (hope and support) and dependent variable (resilience).

Model also shows that coefficients of regression:

$\mathrm{a}_{0}=0.853084$

$\mathrm{a}_{1}=0.316731$

$a_{2}=0.347652$

Since coefficient of regression for both hope and support are positive indicating that hope and support positively influence resilience.

\section{DISCUSSION}

The main goal of this study were to examine if higher level social support and hope were associated with the higher level of resilience in cancer patients and also to determine if resilience mediated the relationship between social support and hope. The finding supported the hypothesis that higher the level of hope and social support will affect the level of resilience of cancer patient but the hypothesis that there will be significance between the interaction of the hope social support and resilience is rejected. There was no interaction effect of high hope and low social support on the resilience of the cancer patient which can be seen in the findings that resilience can buffer the impact of major life stressors such as cancer on the emotional well being [23-24]. This study was able to confirm the hypothesis that higher level of social support affects the level of resilience in the cancer patient this findings is consistent with the previous findings that found that higher level of social support protects the cancer patient from the adverse psychological effects of cancer [25-27].

We reject the hypothesis that there is no interactional relationship between hope and social support on the resilience of the cancer patient. As the interactional relationship between social support and hope was not significant $(p=$ 0.6325). The hypothesis that higher level of social support will affect the level of resilience is also proved in the study, which shows the effect of social support and hope on the resilience of the patient. When there is an increase in the responsibility of the relatives and friends to support the patient suffering from cancer. This confirms the findings of previous studies [3-4] that identified hope and resilience impacting family members' ability to provide care. This study justifies further examination of the concepts of hope and resilience among persons newly diagnosed with cancer and a family member 
and effect of social support. The results of this study can be used to provide a foundation to develop appropriate, valued, non medical interventions.

\section{REFERENCES}

1. Schmer C, Ward-Smith P, Latham S, Salacz M. When a family member has a primary malignant brain tumor: the caregiver perspective. J Neurosci Nursing 2008;40(2):78-84.

2. Twombly R. What's in a name: who is a cancer survivor? J Natl Cancer Inst 2004;96(19): 1414-5.

3. Daly B, Foley H, Douglas S, Lipson AR, Liou C. Similarities and differences in patient and family caregiver quality of life during the first three months of cancer treatment. Oncol Nurs Forum 2007;34(1):206-9.

4. Chen ML. Pain and hope in patients with cancer: A role for cognition. Cancer Nurs 2003; 26:61-7.

5. Lin HR, Bauer-Wu SM. Psycho-spiritual well-being in patientswith advanced cancer: An integrative review of the literature. J Adv Nurs 2003;44:69-80.

6. Lin CC, Tsai HF, Chiou JF, Lai YH, Kao CC, Tsou TS. Changes in levels of hope after diagnostic disclosure among Taiwanese patients with cancer. Cancer Nurs 2003;4:155-60.

7. Wang CE. Developing a concept of hope from a human science perspective. Nurs Sci Quart 2000;13:248-51.

8. Young-Brockopp D. Cancer patients' perceptions of live psychosocial needs. Oncol Nurs Forum 1982;9(4):31-5.

9. Buckley J, Herth K. Fostering hope in terminally ill patients. Nurs Standard $2004 ; 19(10)$ : 33-41.

10. Ebright PR, Lyon B. Understanding hope and factors that enhance hope in women with breast cancer. Oncol Nurs Forum 2002;29:561-8.

11. Lutgendorf SK, De Geest K, Dahmoush L. Social isolation is associated with elevated tumor norepinephrine in ovarian carcinoma patients. Brain Behav Immun 2011;25(2):250-5.

12. Lutgendorf SK, Johnsen EL, Cooper B, Anderson B, Sorosky JI, Buller RE, Sood AK. Cancer 2002;95(4):808-15.

13. McCubbin M, Balling $K$, Possin P, Frierdich S, Bryne B. Family Resiliency in Childhood Cancer. Fam Relat 2002;51:103-11.

14. Moadel A, Morgan C, Fatone A, Grennan J, Carter J, Laruffa G. Seeking meaning and hope: Self-reported spiritual and existential needs among an ethnically-diverse cancer patient population. PsychoOncology 1999;8:378-85.

15. Rustoen T, Wiklund I. Hope in newly diagnosed patients with cancer. Cancer Nurs $2000 ; 23: 214-9$.

16. Young-Brockopp D. Cancer patients' perceptions of live psychosocial needs. Oncol Nurs Forum 1982;9(4):31-5.

17. Rowland $\mathrm{JH}$, Baker $\mathrm{F}$. Introduction: resilience of cancer survivors across the lifespan. Cancer 2008;104(S11):2543-8.

18. Herth KA, Cutcliffe JR. The concept of hope in nursing 3: Hope and palliative care nursing. Br J Nursing 2002;11:977-83.

19. Herth $\mathrm{K}$. The relationship between level of hope and level of coping response and other variables in patients with cancer. Oncol Nurs Forum 1989;16:67-72.

20. Herth K. Abbreviated instrument to measure hope: development and psychometric evaluation. J Adv Nurs 1992;17(10):1251-9.

21. Smith BW, Dalen J, Wiggins K, Tooley E, Christopher P, Bernard J. The brief resilience scale: assessing the ability to bounce back. Int J Behav Med 2008;15(3):194-200.

22. Zimet GD, Dahlem NW, Zimet SG, Farley GK. The multidimensional scale of perceived social support. J Personal Assess 1988;52(1):30-41.

23. Herth, K. (2001). Development and implementation of a hope intervention program. Oncology Nursing Forum, 28, 1009-1017. 
24. Zebrack BJ. Psychological, social, and behavioral issues for young adults with cancer. Cancer 2011;117(S10):2289-94.

25. Cha K, Kim K. Impact of uncertainty on resilience in cancer patients. Asian Oncol Nurs $2012 ; 12(2): 139-46$.

26. Jahn AL, Herman L, Schuster J, Naik A, Moye J. Distress and resilience after cancer in veterans. Res Human Dev 2012;9(3):229-47.

27. Deshields T, Tibbs T, Fan MY, Taylor M. Differences in patterns of depression after treatment for breast cancer. Psycho-Oncology 2006;15(5):398-406.

Acknowledgements - Nil

Conflict of Interest - Nil

Funding - Nil.

\section{THANK YOU TO ALL OUR REVIEWERS - 2014}

A journal is able to maintain its standard and level of publishing due to its reviewers who guide the editor in many ways with regard to acceptance and rejection of manuscripts. The reviewer's job is a painstaking one and is a lot of time is devoted to enhance scientific publishing at no personal gain to the reviewer.

Indian Journal of Mental Health wishes to thank its reviewers for the year 2014

$$
\begin{gathered}
\text { Dr. Nilesh Shah (Mumbai) } \\
\text { Dr. Avinash De Sousa (Mumbai) } \\
\text { Dr. Cicilia Chettiar (Mumbai) } \\
\text { Dr. Sushma Sonavane (Mumbai) } \\
\text { Dr. R. Parmeswaran (Coimbatore) } \\
\text { Mrs. Nadi Jalali (Mumbai) } \\
\text { Mrs. Anuja Deshpande (Mumbai) }
\end{gathered}
$$

